\title{
Using Open Source to realise an NGOSS Proof of concept
}

C.R. Gallen, J. S. Reeve

University of Southampton

Hampshire, England

[cg02r, jsr]@ecs.soton.ac.uk

\section{Abstract}

This paper discusses the aims, objectives and early deliverables from the OpenOSS project which has been set up with the sponsorship of a number of Telecommunications Service Providers to investigate the potential value of open source as an enabler for rapid innovation within the telecommunications industry. We then discuss how the OpenOSS toolbox is being applied to help in the specification of next generation network management solutions for Digital TV networks

\section{Keywords:}

Open Source, NGOSS, OSS/J, Action Research, OSS, Operations Support Systems, Systems Integration, Requirements Management. 


\section{OpenOSS}

- University backed industrial research

- Working with the Telemanagement Forum

- Action Research (i.e participate in projects )

- Investigate the benefits of NGOSS specified solutions

- Investigate the implications of open source realisations - Proofs of concept

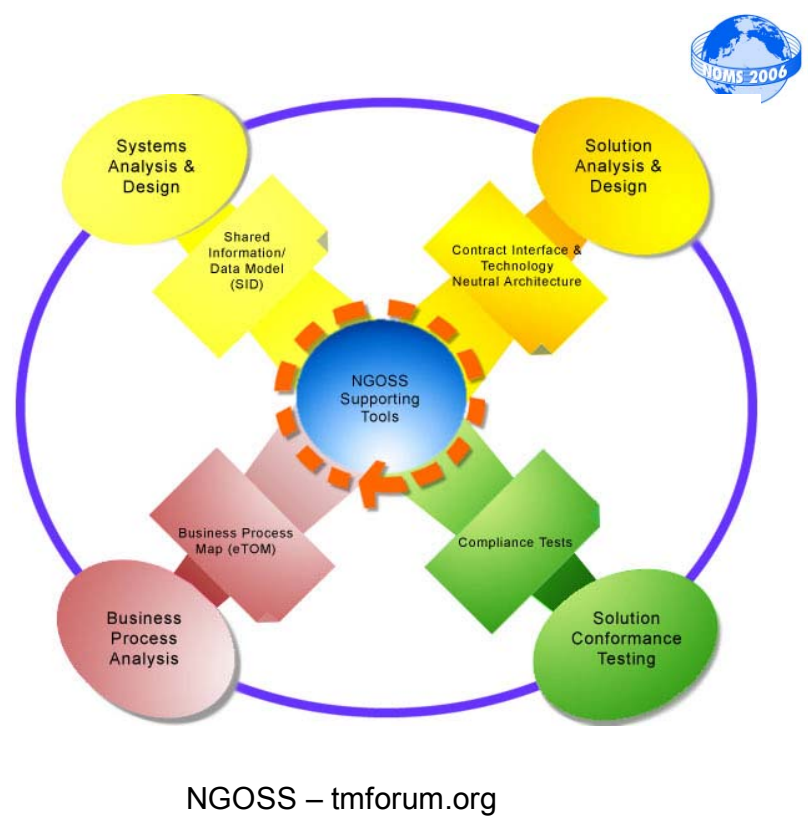

Caing Galen Lnivesis of

The University of Southampton has initiated within the Telemanagement Forum[2] a cross industry Action Research project called OpenOSS[1]. This is investigating novel ways of using Open Source software to accelerate the adoption of next generation management standards for Operational Support Solutions.

Systems implemented in open source potentially allow for more rapid prototyping that would be possible with conventional COTS products. This allows proofs of concept to be developed in an open and non competitive environment before commercial decisions need to be made on the choice of architecture.

By delivering an open test bed this project hopes to provide a new and complementary route to expedite the testing, development and realisation of Telemanagement Forum standards. The research is focusing on the problems and benefits of using NGOSS as a tool for integrating a solution consisting of NGOSS components. To support this work, a number of open source enterprise management solutions are being enhanced to allow them to be viewed as simple NGOSS components.

A key standard being investigated by the project is the New Generation Operations Support Systems (NGOSS) proposed by the Telemanagement Forum[3]. This is a model based, technology neutral standard designed to help service providers specify their network management solutions for next generation networks. Having been developed for over 10 years, NGOSS is beginning to be adopted by the industry and we are seeking to use this research to quantify how NGOSS provides value and to further its deployment as a mainstream standard

It should be understood that the use of NGOSS as a tool for specifying a system does not force the use of 'NGOSS components'. The primary use of NGOSS in this context is as a tool to assist with the integration of existing telecoms management components into a complete system.

The NGOSS standard takes account of the business processes and the information models used by the service provider but does not tie the designer to a specific implementation technology until the later stages of design or procurement. It is an example of a model driven approach to component system design targeted specifically at the telecoms market. 


\section{Research Approach}

- Action Research

- Contribute to industry

knowledge on how to use these technologies

- Quantify the business benefits they offer

- Contribute back from our experiences into the evolution of the standards

- Promote tools or methodologies for practically working with NGOSS and related technologies

- Open Source

- Leverage the work of other groups

- Contribute work back - such that it can be built upon and

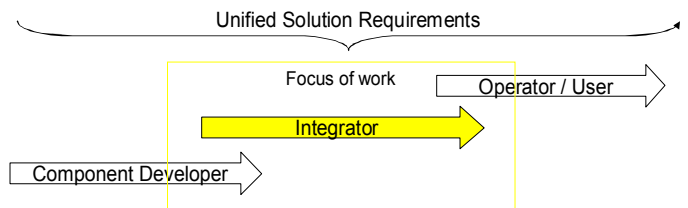
not lost

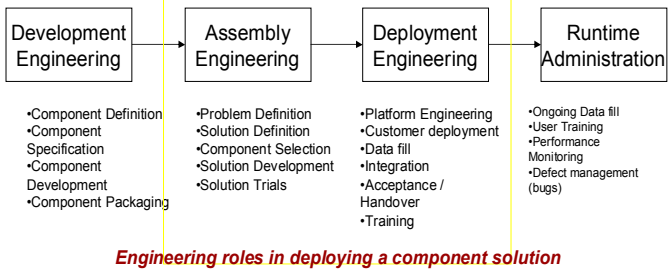

As described above, the research approach being used is Action Research. This research paradigm involves generating original knowledge in the context of participating in a real project with working practitioners. The hypothesis being that much more can be learnt about the practical use of a technology in a realistic setting than in an artificial lab environment.

Action Research requires access to a number of real world business contexts in which to undertake investigations. A number of companies including BT, Vodafone, C\&W, COLT, Agilent, Automagic and Invocom have been participating in this work.

NGOSS and its related technologies such as OSS/J have been defined through strategic R\&D participation by many organisations over a number of years but are only now beginning to be taken into daily use in the market place. Through our research we want to;

- Contribute to industry knowledge on how to use these technologies

- Quantify the business benefits they offer

- Contribute back from our experiences into the evolution of the standards

- Promote tools or methodologies for practically working with NGOSS and related technologies

In addition, there is a gathering awareness that Open Source software offers some unique opportunities for more efficient definition of operational support solutions. We want to quantify what this means. In order to do this we are using open source software and participating in open source development in order to have a close understanding of that the open source development paradigm can mean. This is particularly relevant in the prototyping stage of new projects.

By combining NGOSS with open source, we are trying to see if we can prototype NGOSS solutions without commitment to the final implementation technology and use the resulting NGOSS Technology Neutral design to drive the procurement process for COTS components. 


\section{Telemanagement World May 2005 OpenOSS Catalyst}

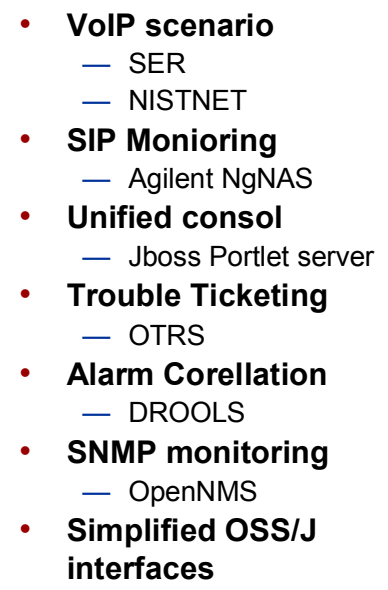

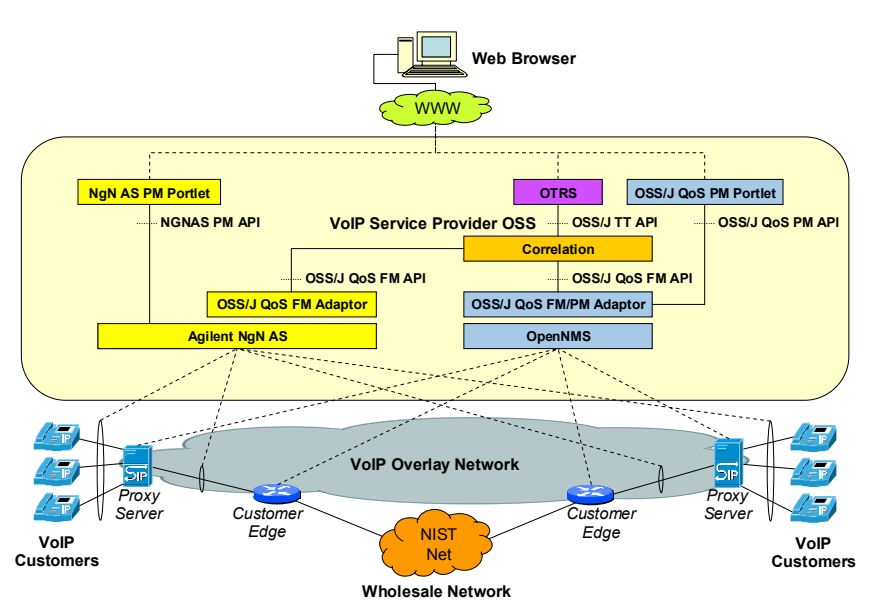

NOMS06 - 1

A number of partners contributed to the OpenOSS catalyst demonstrated at Nice Telemanagement world in May 2005. This combined a number of open source and COTS components to build a proof of concept. The key benefit from this work was in proving that open source components could be used to illustrate NGOSS solutions. Our experience of the Catalyst is summarized below;

Open source

- Open source approach generated significant interest and good working relationships

- Suitable open source software available

- Good support from open source software providers

- Lack of experience on the selected open source software

- Building software repository and development tools

- Developing experience and best practices

OSS/J

- Able to use the OSS/J APIs straight away

- Integration of OSS/J Reference Implementations required more effort than first thought NGOSS

- Excellent framework for understanding the problem domain and solution requirements

- Did not follow the NGOSS lifecycle, this time!

- NGOSS pre-populated tools would be very helpful

Catalyst experience

- Excellent way to focus effort

- A good vehicle for a proof-of-concept

- Provides a great way to get visibility for the project and encourage further participation 


\section{Using A Testbed in the Design Process}

- How do we use NGOSS to design real solutions?

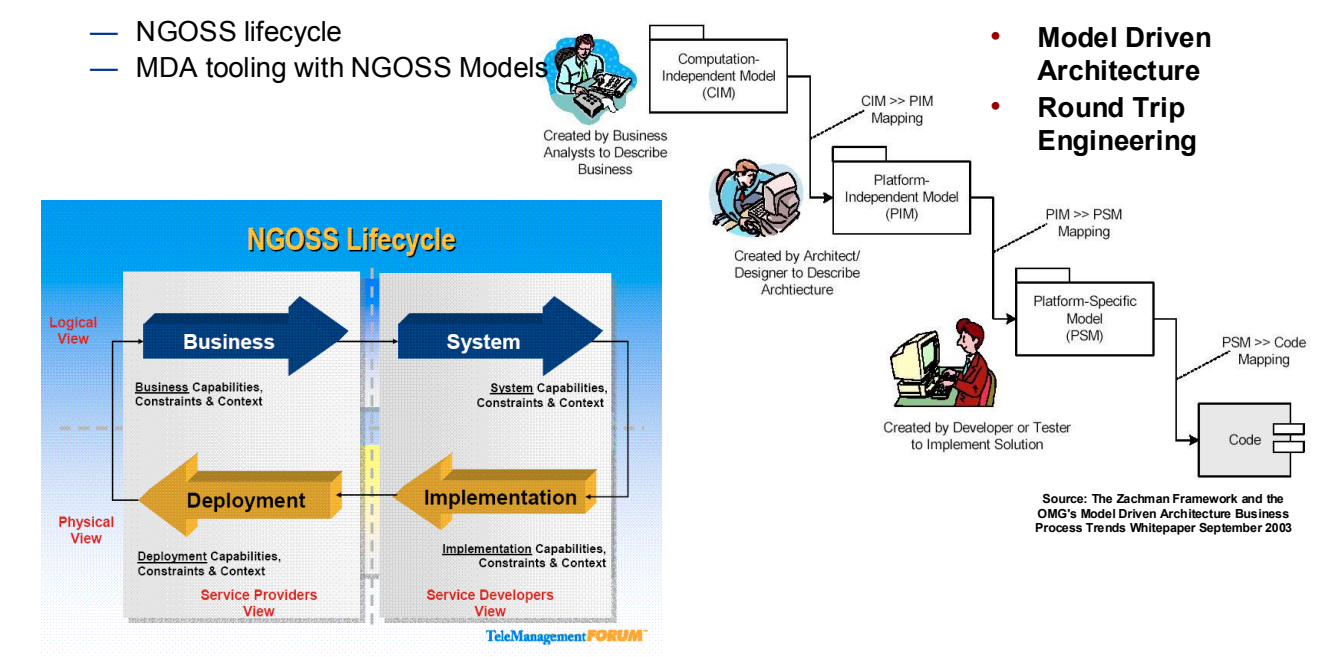

Having created some useful components, we are now extending the work to begin using the NGOSS design process. Our approach is to find real world projects in which we can begin using NGOSS to assist with the design cycle. Later in this paper we will discuss a Digital TV project which is being used as a vehicle for our research but first we discuss how NGOSS can help with design.

The NGOSS Lifecycle allows the business and system requirements for a project be considered in a Technology Neutral Model realised in UML. The business and system views provide a specification for implementation in the chosen technology. The implementation view is concerned with the technologies used to implement the design and the deployment view is concerned with how the system is actually deployed.

Central to NGOSS is the Shared Information/Data Model (SID) [4] which is built from a number of industry standard information models for understanding the relationships between systems within a network. Work is ongoing to show how a complimentary standard, the DMTF CIM can inter work with NGOSS. We believe that in practice many designs will have to mix and match the CIM and SID at the resource modelling layer.

As already stated, NGOSS provides the opportunity to start with a consistent technology neutral specification in UML allowing a customer to work the design issues with their suppliers using UML in an industry standard framework. Rather than dealing with implementation specific UML which is tied to the potential supplier's systems, NGOSS allows the initial design specification to be created using a standard model which is then mapped onto the vendor's application.

This relates very closely to the OMG Model Driven Architecture approach to design [5] and a number of MDA tools are becoming available to help with NGOSS modelling. It should be noted that while we believe it will be some time before a full round trip design of an NGOSS system can be done with these systems, UML tooling can greatly assist with developing an NGOSS blueprint for a solution. One such tool is being developed by Automagic [6] which is owned by a former director of the NGOSS program in the Telemanagement Forum. This is based upon a widely available and relatively inexpensive MDA tool called Enterprise Architect [7]. The models created in this tool can be easily exported to other UML tools using XMI. 


\section{NGOSS and OpenOSS in Design Process}

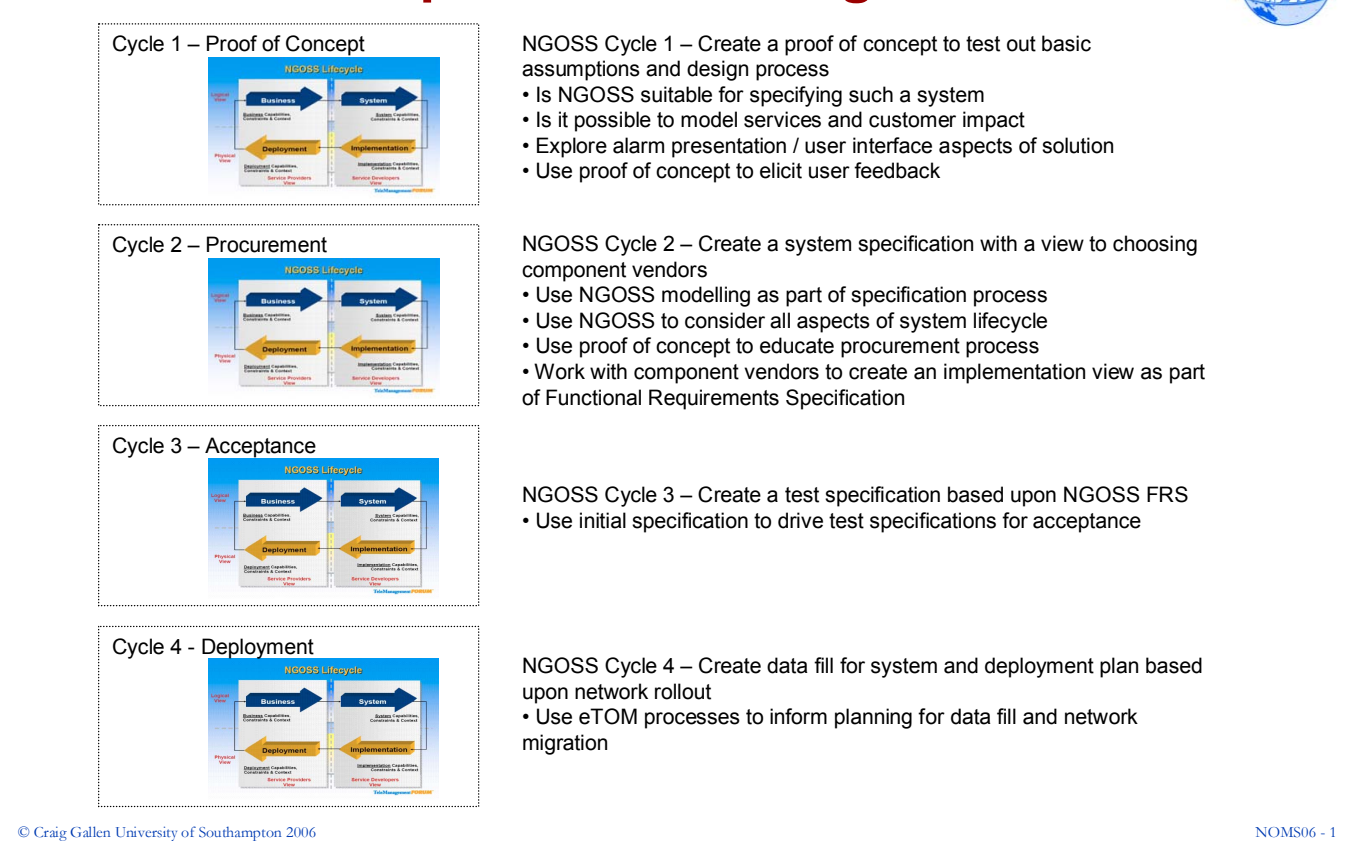

The slide above illustrates how the NGOSS lifecycle would be used to prototype a design and prove it out throughout the procurement process.

- Cycle 1 - Initial proof of concept

A simple proof of concept would be created using a mixture of open source and pre-production software which provided a simple technology specific implementation of the specification in order to prove the concept. This would not be the final solution but would provide sufficient proof points to assure the customer that the solution could be realised through COTS solutions. it would also serve to educate all of the potential stakeholders as to the possibilities and requirements for the solution.

- Cycle 2 -Design Tendering Process

Use initial technology neutral design to engage with suppliers for final system and evaluate collected responses.

- Cycle 3/ 4 - Acceptance and Deployment

Use design for acceptance testing. If done correctly, the proof of concept can provide an acceptance test harness for the system.

We have been fortunate enough to be able to try out this process by participating in the initial proof of concept stage for the design for the UK digital TV roll out. 


\section{Modelling NGOSS Contracts}

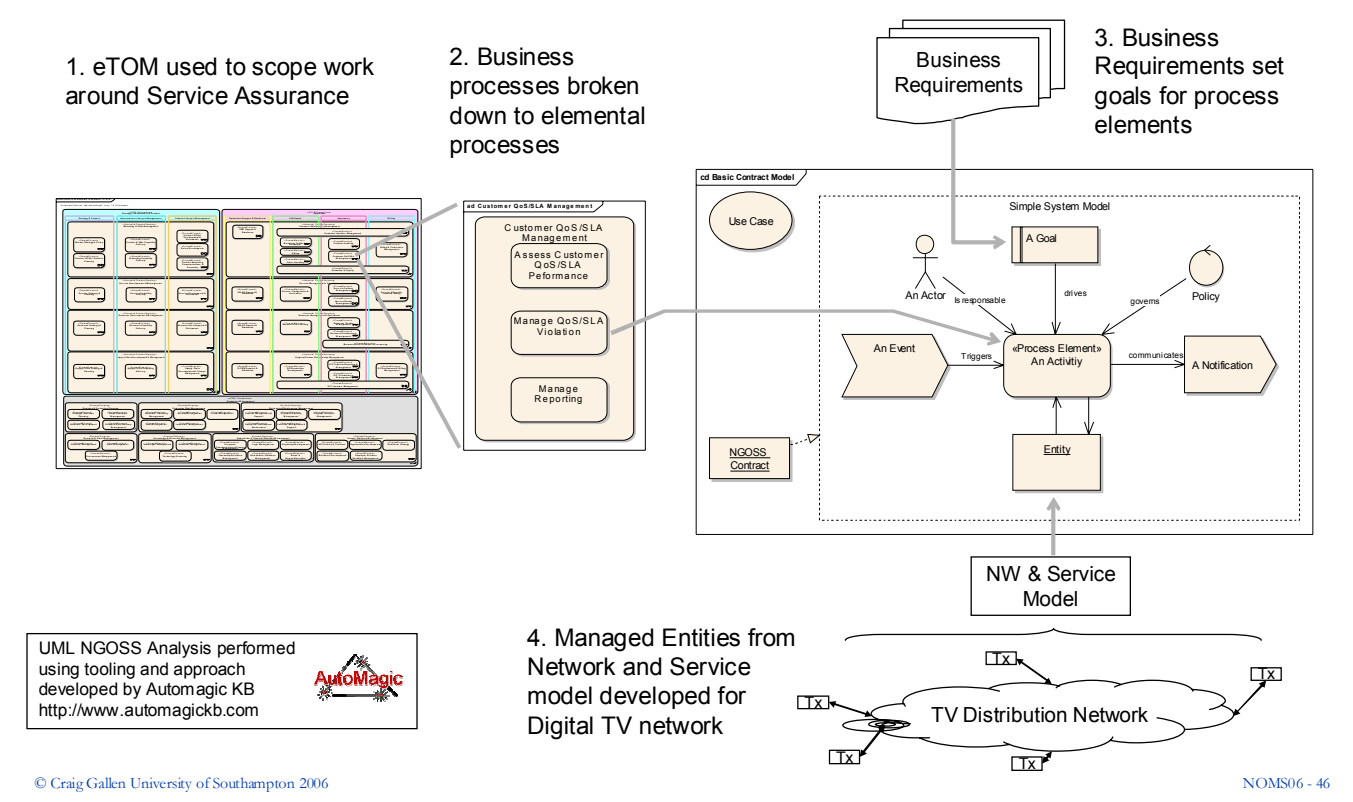

The function of a component in an NGOSS system is described by the NGOSS contract. The exact formal definition of an NGOSS contract is still being developed. However, Automagic have proposed tooling which can help in capturing in a single UML based model all of the information pertaining to a contract. We are using this methodology in our work. This is illustrated above

NGOSS provides a framework called the extended Telecoms Operations Map (eTOM)[8] to model the business processes within a service provider. The eTOM allows service providers and vendors to share a common understanding of their internal businesses processes and to investigate how the operations support systems will interact with the human activities and organisational structure they support.

The eTOM has been recognised as an ITU-T standard (M.3050) and can help with planning a significant technology migration because it provides a framework to consider and plan for all of the systems and processes which have to change as the network is being rolled out. In the Digital TV project,

- Operations Support and Readiness process is concerned with the deployment, data-fill, and user training associated with introducing the new systems in each region.

- Fulfilment is concerned with the actual migration of customer from UHF to Digital transmission.

- Assurance is concerned with customer SLA's through the migration.

As an integral part of NGOSS, the eTOM allows us to consider not just the systems themselves but also the cost and process for introduction in the context of the overall digital migration project.

In our analysis, we use the eTOM to break the business requirements down into atomic process elements which then are used to set the scope of each contract. Working from the top down, we analyse the impact of required service SLA's against existing business processes. Working from the bottom up, we analyse the data available from the network and design policies for translating this data into meaningful business information. 


\section{NGOSS prototype of UK Digital TV Service}

- Background

- Digital TV rollout begins in UK within 5 years

- Digital TV convergence with Mobile

- Opportunity for broadcasters

- Analyse the Digital TV network management problem

- De-risking the Digital TV management strategy and procurement process

- Leveraging emerging mainstream Telecommunications management technologies

- Develop a solution against a strategic understanding of Telecoms/Digital TV convergence rather than architecting a solution which mirrors their current business

- Considering business process analysis as integral to the network management design

- Future-proof the architecture for managing next generation services

- Opportunity for OpenOss

- Add novelty to the research by extending standards originally intended for telecoms management towards the management of Digital TV.

- Provide a realistic project which is right-sized for the research resources and which has a degree of commercial support.

Plans are in place for switching off UHF television for the in the United Kingdom. The transmission service providers wish to investigate the various options open to them for managing the next generation TV services in order to given them confidence in establishing a strategic direction prior to placing contracts for the provision of management solutions. Involving OpenOSS in this work provides the following benefits;

For The broadcasters

- Supplementing their resources to analyse the Digital TV network management problem

- De-risking the Digital TV management strategy and procurement process

- Leveraging emerging mainstream Telecommunications management technologies

- Develop a solution against a strategic understanding of Telecoms/Digital TV convergence rather than architecting a solution which mirrors their current business

- Considering business process analysis as integral to the network management design

- Future-proof the architecture for managing next generation services

For University of Southampton Research (OpenOSS)

- Providing a local, accessible environment against which to investigate the use of next generation management specification standards

- Add novelty to the research by extending standards originally intended for telecoms management towards the management of Digital TV.

- Provide a realistic project which is right-sized for the research resources and which has a degree of commercial support. 


\section{Digital TV Service Assurance Problem}

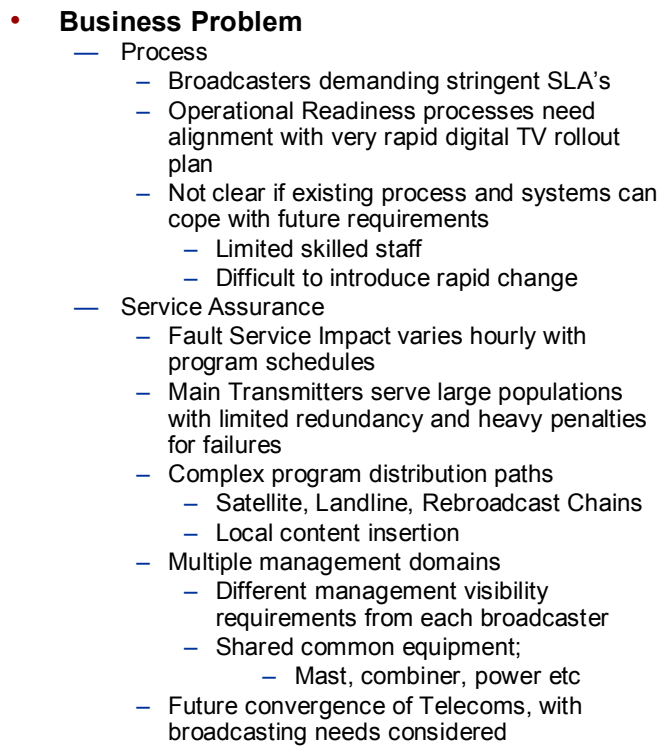

- Technical Problem

- Limited industry agreement on network management standards.

- No proven large scale Digital TV management solution

- Choice of Transmitter Control protocols

- Currently use proprietary SCADA

- Opportunity to leverage standards - SNMP, WBEM, Web Services, Others?

- No common Network and Service models for Digital TV.

- Is it possible to leverage

- DMTF CIM Vs NGOSS SID

- ETSITR 101290 v1.2.1 Broadcast

DTV measurement spec \& MIB

- Requirement for policy/ rule driven Service assurance

- Can we exploit a rapid solution development approach using open source to prototype a solution

The UK has enjoyed digital TV for a number of years as a supplemental service to the UHF TV service. However starting in 2012, the UHF TV service will be turned off and replaced with a significantly enhanced digital TV service having $99 \%$ UK coverage and a much wider selection of channels. This poses both business and technical problems to be resolved.

In the UK, the transmission networks are separately owned and provide services to the television broadcasters. The broadcasters are placing major new demands on the transmission service providers as they re-negotiate contacts for the digital service rollout. This will require a significant rethink in the way in which the transmission service providers conduct their business.

Telecoms service management has traditionally been geared towards large volumes of small subscribers and a highly redundant network. In broadcasting a relatively small number of stations serve very large populations and so the revenue impact of any fault can be much greater than in telecoms. The impact also varies with the program schedules. With a much larger number of services being carried by common equipment, and each broadcaster wanting sophisticated availability reporting, the problem of running a digital TV business will be more complex than at present

Technically, the switch to digital TV also poses a number of management problems. For the last 20 years, the transmission service providers have relied upon SCADA protocols to manage their networks. However transmitter manufacturers are now proposing that they use SNMP for both monitoring and control. The service providers are very unclear as to the potential impact of this proposition. Each transmitter manufacturer is proposing their own proprietary MIB and there are no common standards to draw upon. Further more little if any work has been done to model a large scale digital TV network in order to do service impact analysis.

The service providers want to understand what value telecoms models such as the SID (or the DMTF CIM) could provide as a basis for resource management and service impact analysis. They also want to understand the choice of protocols available for management communication.

Given the tight timescales it would not be appropriate to do a purely theoretical study and so the service providers welcome the opportunity to rapidly prototype a solution in order to get a first order understanding of the issues involved. 


\section{Digital TV Service Assurance}

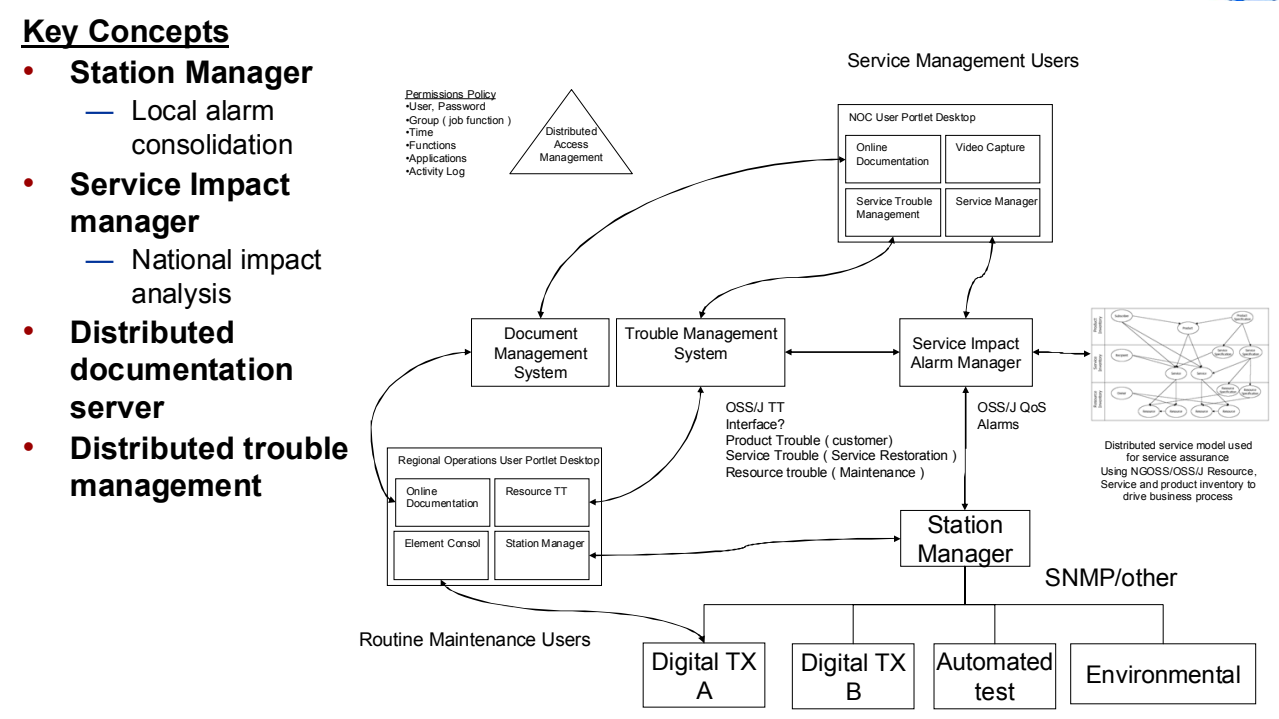

The slide above illustrates a very simplified view of the management topology for a digital TV network. Over 1000 stations would have their own software 'Station Managers' which would communicate with a centralised alarm manager. Service impacting alarms would be forwarded to the Service management centre while fault alarms would be forwarded to the mobile maintenance teams for repair.

The trouble management process within the old UHF network relied upon the expertise of the operator to attach meaning to the criticality of an alarm. Mimic diagrams and priority ordered lists based upon station importance etc were used to help prioritise and guide the operator to an appropriate action. However the new trouble management process will be much more complex and the network operators will require much more help to prioritise any work to fix network faults.

- Many services having different business priorities are carried by the same network elements

- Network element configuration will significantly change their behaviour

- Fault impact will be much more time dependant and difficult to predict. High value 'Advertising breaks' are not synchronous across the many channels.

NGOSS allows operators to consider their trouble management in a unified way at several levels;

- Resource trouble management - which box is in alarm? Do we need to fix it immediately or can it wait. What tasks are required on site?

- Service Trouble management - which services are affected by this equipment fault. Does it matter? Is there a fix to restore the service

- Product trouble management - which customers are affected, which SLA's are violated, what is the financial implication? How do we communicate the status?

Research is needed to determine the best way to manage network trouble and present relevant information to human operators in the trouble management process. 


\section{Digital TV Proof of Concept}
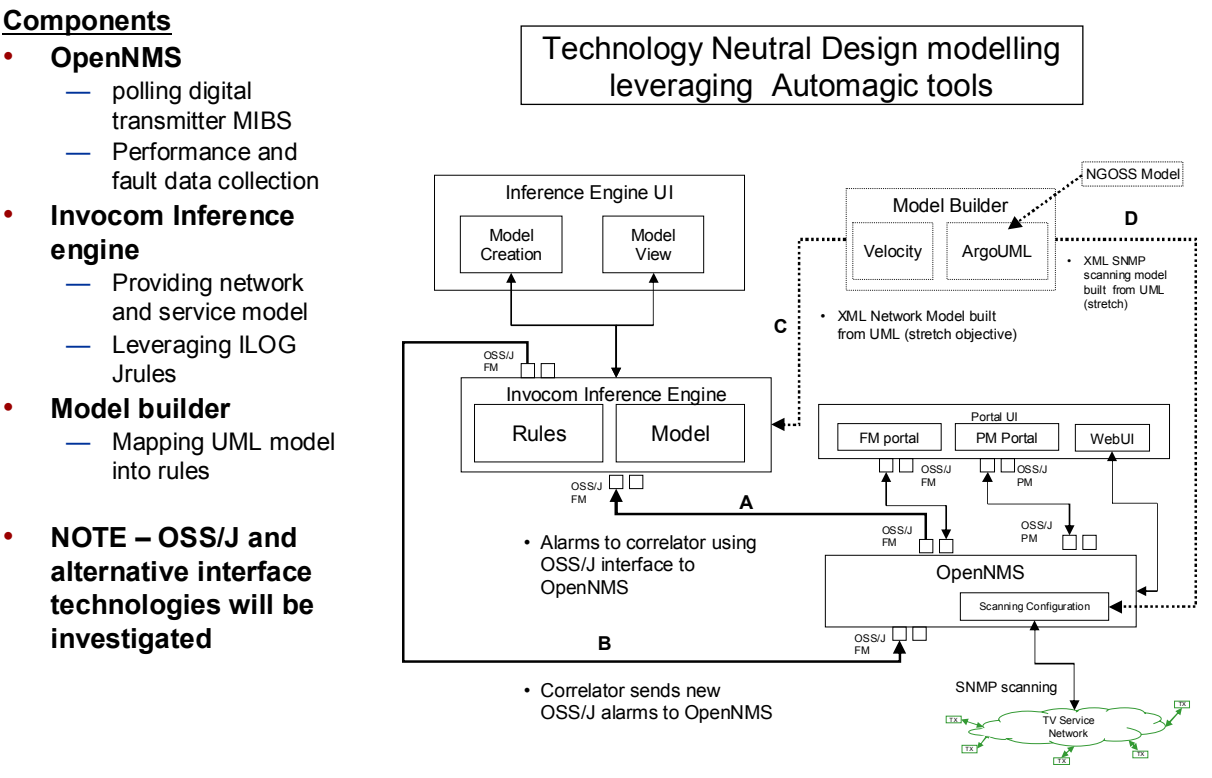

echnology Neutral Design modelling leveraging Automagic tools

The slide above illustrates the Initial Broadcast TV proof of concept project. The key value of this exercise will be in developing a system specification prior to choosing which components to use. The focus of the work will be on modelling the network and services and investigating SNMP and alternative protocols for management.

It should be noted that the focus of this work is on Service Assurance. Clearly, the other management areas (Configuration, Security etc) will need investigated - but this is beyond the scope of the current project.

The basic requirement is to explore how an NGOSS approach to OSS would be applied to managing a transmitter network and in particular whether a storm of SNMP traps can be reduced to a manageable set of meaningful alarms using trap to alarm mapping (through OpenNMS) and a correlation engine with knowledge of the network. The correlation engine will use ILOG Jrules along with a proprietary alarm modelling technology from Invocom which will be interconnected with OpenNMS using and OSS/J Qos FM interface.

In this diagram, Open NMS will map SNMP events to OSS/J alarms using an XML driven mapping. The OSS/J Qos interface labelled 'A' sends X773 alarm events to the correlator and can be polled for the current alarm list. The OSS/J Qos Interface labelled 'B' allows the correlator to send root cause alarm events (as a result of correlation) back to OpenNMS 


\section{Summary and Conclusions}

\section{- OpenOss}

— Is providing a vehicle for university research to tackle real world management problems

- Rapid application development backed by a management model provides a means to do proof of concept investigations in a disciplined and structured process

- The Digital TV management proof of concept

— Is significantly de-risking the deployment of digital TV in the UK by identifying early problems and developing the management architecture before major purchasing decisions are made.

\section{- Flexible approach to NGOSS}

- By choosing to investigate real world problems, we are taking a flexible approach to NGOSS. This mirrors what will happen in any real world engineering situation.

- Our pragmatic approach to modelling is using the bits of NGOSS which work for us and combining them with other work such as the DMTF CIM in order to deliver time to market solutions.

- This approach makes NGOSS tractable to the Systems Integrator.

In conclusion, the use of NGOSS as part of the network management design provides the following business benefits;

- Considering the organisation process issues at same time as designing systems

- Designing a solution which can be extended to other telecoms management problems and can also integrate with partners' processes. Considers the issues of planning network deployment and customer migration at the same time as system design

- Systematically trials different operations methodologies and organisation models without prejudicing the overall system design process.

While some may be zealous about methodological purity, our approach to NGOSS has been to try and make it practical and useful to the average engineer by proving out tooling and methodology in real world situations. The use of open source components allows us to rapidly prototype solutions which prove out the technology neutral design as it is developed. The components as well as the design models can then be passed on to the next stage of the project as more complete artefacts than simple paperwork.

We believe this approach will promote the uptake of NGOSS and more rapid network management solution development by the telecoms industry.

[1] (2005) OpenOSS Initiative www.openossinitiative.org/

[2] (2005) Telemanagement Forum www.tmforum.org

[3] Telemanagement Forum (2004) NGOSS Overview http://www.tmforum.org/browse.asp?catID=1912

[4] (2005) NGOSS Shared Information/Data Model (SID) http://www.tmforum.org/browse.asp?catID=1684

[5] (2005) OMG Model Driven Architecture (MDA) http://www.omg.org/mda/

[6] (2005) AutoMagicKB: Knowledge-Based Intelligent Solutions for Industry http://www.automagickb.com:8080/AutoMagicKB

[7] (2005) Enterprise Architect http://www.sparxsystems.com.au/

[8] (2005) eTOM Overview http://www.tmforum.org/browse.asp?catID=1648 